\title{
ANNUAL INFLUENZA VACCINATION EFFECT ON THE SUSCEPTIBILITY TO COVID-19 INFECTION
}

\author{
Betul Erismis ${ }^{1,2}$, Semsi Nur Karabela ${ }^{2,3}$, Fatma Eksi $^{4}$, Faruk Karandere ${ }^{1}$, Bekir Dogan ${ }^{1}$, Fatih Okay ${ }^{1}$, Melih Filiz', \\ Hakan Kocoglu' ${ }^{1}$, Halim Issever ${ }^{5}$, Mehmet Hursitoglu ${ }^{1,6}{ }^{1}$, Kadriye Kart Yasar ${ }^{3}$ \\ ${ }^{1}$ Internal Medicine Department, Bakirkoy Dr. Sadi Konuk Training and Research Hospital, University of Health Sciences, Istanbul, Turkey \\ ${ }^{2}$ Hamidiye Health Services College, University of Health Sciences, Istanbul, Turkey \\ ${ }^{3}$ Infectious Diseases Department, Bakirkoy Dr. Sadi Konuk Training and Research Hospital, University of Health Sciences, Istanbul, Turkey \\ ${ }^{4}$ Infectious Diseases Department, Bagcilar Training and Research Hospital, University of Health Sciences, Istanbul, Turkey \\ ${ }^{5}$ Public Health Department, Istanbul Medical Faculty, Istanbul University, Istanbul, Turkey \\ ${ }^{6}$ Internal Medicine Department, Basaksehir Cam and Sakura Sehir Hospital, University of Health Sciences, Istanbul, Turkey
}

\section{SUMMARY}

Objectives: We aimed to study the effect of seasonal influenza (flu) vaccination on the susceptibility to coronavirus disease 2019 (COVID-19).

Methods: A total of 203 healthcare workers of a pandemic centre of Istanbul, Turkey, were included in this retrospective study. According to the presence or absence of flu vaccination, participants were divided into group 1 and group 2. A comparison of the rate of COVID-19 was done between these two groups. Also, the mean age and the sex ratio of females/males were evaluated and compared between these two groups.

Results: Group 1 participants $(n=65)$ were older than participants in group $2(n=138)(p<0.05)$. Despite of this, interestingly, the COVID-19 infection rate was lower in the 1 st group (in comparison to the 2 nd group) $(p<0.05)$.

Conclusion: Our study results showed that, even if low, the flu vaccination may have a protective effect on the susceptibility to COVID-19 infection. Using this beneficial adjuvant effect of the vaccine may help us in this unpredictable battle with the COVID-19 pandemic. Further studies are needed to confirm this assumption.

Key words: influenza, vaccination, COVID-19, protection

Address for correspondence: M. Hursitoglu, Internal Medicine Department, Bakirkoy Dr. Sadi Konuk Training and Research Hospital, University of Health Sciences, Istanbul, Turkey. E-mail: hursitoglum@yahoo.com

https://doi.org/10.21101/cejph.a6573

\section{INTRODUCTION}

Seasonal influenza is an acute respiratory illness caused by influenza A or B viruses. It causes an annual epidemic. To decrease its spread, annual influenza vaccination is advised especially in high-risk groups. Healthcare workers are within these risk groups (1).

Coronavirus disease 2019 (COVID-19) is caused by the new coronavirus (SARS-CoV-2). It started in December 2019 in China (2). Then it spread to most parts of the world. In Turkey, the first COVID-19 positive case was reported on March 10, 2020 (3). Later, it had taken a pandemic manner. Turkish hospitals and our centre especially (Bakirkoy Dr. Sadi Konuk Training and Research Hospital, Istanbul, Turkey) reacted speedily against this infection outbreak. So, this centre became one of the biggest pandemic centres for combating COVID-19 infection in Istanbul, Turkey. Some researchers hypothesize that flu vaccination may foster immunity against COVID-19 (4). Some researchers found lower mortality of COVID-19 infection in persons who had an influenza vaccination (5). Also, a study from Brazil involving over 92,000 patients with COVID-19 showed that vaccination had reduced the severity and death of this disease (6). As far as we know, there is no study about the effect of flu vaccination on the susceptibility to COVID-19 infection. So, we aimed to study this effect.

\section{MATERIALS AND METHODS}

This study was approved by the Bakirkoy Dr. Sadi Konuk Training and Research Hospital Ethical Committee and written consent was obtained from the participants. The participants consisted of the abovementioned hospital healthcare workers (medical and paramedical) who were actively working at the pandemic clinics (Infectious Diseases and Internal Medicine Clinic). These clinics were chosen to make exposure of the participants to the COVID-19 virus standardized. Those who accepted to participate were given a questionnaire containing the following data: age of the participant, gender, place of work, history of influenza vaccination before or during COVID-19 pandemic (yes/no), history of Covid-19 infection, permission to reach the person's clinical data from the hospital's record. 
The participants' given data were checked from "E-nabiz" system of the Turkish Ministry of Health. This system includes person's previous and present medical records*. A total of 203 participants were included in the study. According to the presence or absence of this year influenza vaccination, the participants were divided into group 1 (vaccinated group, $n=65$ ) and group 2 (non-vaccinated group, $n=138$ ). The following data of the participants were recorded and analysed: age, gender, chest CT findings (COVID-19 infected persons), and laboratory parameters (COVID-19 infected persons).

The primary aim of this study was to see and compare the COVID-19 rate in the abovementioned two study groups. The secondary aim was to compare other parameters between the two groups and with relation to the presence or absence of COVID-19 infection history, age, gender, and chest CT findings.

\section{Statistical Analysis}

The analysis was performed using the jamovi (7). Description of data was expressed by mean, standard deviation, median, and interquartile range, as appropriate. The distribution of variables was checked with the Kolmogorov-Smirnov test. For a comparison of variables of a normal distribution, the t-test for independent samples was used and the Mann-Whitney $U$ test was used for the comparison of variables with non-normal distribution. A comparison of frequencies was done by chi-square test. For the degree of the correlation, a Phi and Cramer's V value was determined (between 0.0-1.0). A Phi and Cramer's V value close to 0.00 indicates no association. A value $>0.15$ is indicative of a strong, and $>0.25$ is indicative of a very strong association (8). A p-value $<0.05$ was accepted as significant.

\section{RESULTS}

The analysis was done with a total of 203 participants (female/ male ratio was 124/79). The mean $\pm \mathrm{SD}$ (median [min-max]) age of the participants was $35.70 \pm 10.60(33.00$ [19.00-66.00]) years. The comparison of the influenza vaccinated $(n=65)$ with the non-vaccinated $(n=138)$ group is shown in Table 1. Although the ratio of females to males was not different between the two study groups, the non-vaccinated group was significantly younger than the vaccinated one. In addition to that, the rate of COVID-19 infection in the non-vaccinated group was significantly higher than in the vaccinated one (Table 1). There was a negative relation between the influenza vaccination history and COVID-19 infection history: relative risk $=0.834$ (95\% CI: 0.749-0.929). The Phi and Cramer's V coefficient was 0.195. The comparison of 203 participants according to the history of COVID-19 infection is shown in Table 2 . The age and gender were not significantly different between the infected and non-infected group of participants. Additionally, the non-infected participants had no comorbidities.

\section{DISCUSSION}

As known, flu and COVID-19 are different viral diseases. Although there is no definite evidence on this issue, some re-

Table 1. Comparison of participants according to their influenza vaccination status $(N=203)$

\begin{tabular}{|c|c|c|c|c|}
\hline \multirow{2}{*}{\multicolumn{2}{|c|}{ Parameters }} & \multicolumn{2}{|c|}{ Influenza vaccination status } & \multirow{2}{*}{$\mathrm{p}$-value } \\
\hline & & \multirow{2}{*}{$\frac{\text { Vaccinated }(n=65)}{39.1(10.6)}$} & \multirow{2}{*}{$\frac{\text { Not vaccinated }(\mathrm{n}=138)}{34.1(10.3)}$} & \\
\hline \multirow{2}{*}{ Age (years) } & Mean (SD) & & & \multirow{2}{*}{$<0.001$} \\
\hline & Median (min-max) & $40.0(19.0-66.0)$ & $30.0(20.0-66.0)$ & \\
\hline \multirow{2}{*}{ Gender } & Female & $36.0(55.4 \%)$ & $88.0(63.8 \%)$ & \multirow{2}{*}{ NS } \\
\hline & Male & $29.0(44.6 \%)$ & $50.0(36.2 \%)$ & \\
\hline \multirow{2}{*}{ COVID-19 } & Infected & $4.0(6.2 \%)$ & $30.0(21.7 \%)$ & \multirow{2}{*}{0.006} \\
\hline & Not infected & $61.0(93.9 \%)$ & $108.0(78.36 \%)$ & \\
\hline
\end{tabular}

NS - not significant

Table 2. Comparison of COVID-19 infected participants with non-infected peers $(N=203)$

\begin{tabular}{|c|c|c|c|c|}
\hline \multirow{2}{*}{\multicolumn{2}{|c|}{ Parameters }} & \multicolumn{2}{|c|}{ COVID-19 } & \multirow{2}{*}{$\mathrm{p}$-value } \\
\hline & & \multirow{2}{*}{$\frac{\text { COVID-19 (+) }(n=34)}{36.9(10.1)}$} & \multirow{2}{*}{$\begin{array}{c}\text { COVID-19 (-) }(n=169) \\
35.5(10.7)\end{array}$} & \\
\hline \multirow{2}{*}{ Age (years) } & Mean (SD) & & & \multirow{2}{*}{ NS } \\
\hline & Median (min-max) & $38.0(22.0-56.0)$ & $33.0(19.0-66.0)$ & \\
\hline \multirow{2}{*}{ Gender } & Female & $23.0(67.7 \%)$ & $101.0(59.8 \%)$ & \multirow{2}{*}{ NS } \\
\hline & Male & $11.0(32.4 \%)$ & $68.0(40.2 \%)$ & \\
\hline \multirow{2}{*}{ Comorbidities } & Present & $7.0(20.6 \%)$ & 0 & \multirow{2}{*}{$\mathrm{N} / \mathrm{A}$} \\
\hline & Not present & $27.0(79.4 \%)$ & 0 & \\
\hline
\end{tabular}

NS - not significant; N/A - not applicable

\footnotetext{
*https://enabiz.gov.tr/Yardim/Index?lang=en (last checked on 30 July 2020)
} 
searchers clarify that flu vaccination does not affect susceptibility to COVID-19 and insistently recommend flu vaccination (9). Other researchers claim that flu and tuberculosis (TB) infections decrease susceptibility to COVID-19 and its mortality while flu and/or BCG vaccination make a reverse effect on COVID-19 (10). Furthermore, there is a claim of no beneficial effect of vaccination against pneumonia on the susceptibility to COVID-19 (11). So these and alike not approved hypothesis will make the rate of flu vaccination low even in healthcare workers (12). The flu vaccination rate in our healthcare workers - participants was $32.02 \%(65 / 203)$ despite of being in a high-risk group (1). Our study participants have included healthcare workers that are working in COVID-19 infected pandemic services. In other words, they faced the highest risk of exposure to COVID-19 infection at the study hospital. Comparing those who did have a seasonal flu vaccine before the COVID-19 pandemic in Turkey, i.e. before March 2020, (group 1) with those without vaccination (group 2) showed a lower rate of COVID-19 infection in the group 1 of participants $(p=0.006)$. We should mention that besides having a low rate of COVID-19 infection, group 1 participants were significantly older than the other group $(\mathrm{p}<0.05)$ (Table 1). After completion of the study data (on 30 July 2020), further 2 participants from group 2 were infected with COVID-19. When we take this into consideration the p-value changes from 0.006 to 0.003 and the Phi and Cramer's V coefficient value changes from 0.195 to 0.210 , respectively. This low rate of COVID-19 infection in the flu vaccinated healthcare workers is most probably due to the preventive effect of this vaccine against COVID-19 infection. It could not be explained by the protective measures effect(s) (such as masking, hygiene, etc.) because there should not be a great difference in the protective measures between the two study groups (the same working conditions and protective measures). Early life vaccination against TB (i.e. BCG) is mandatory in the Turkish community. Pathak et al. noted in their research that high flu vaccination coverage affects COVID-19 mortality inversely, while both countries with both BCG and flu vaccinations had relatively less mortality as compared to those with flu vaccination alone (10). So, our study finding of beneficial effect of seasonal flu vaccination on decreasing the susceptibility to COVID-19 partially may be due to the presence of BCG vaccination in Turkey. Further studies are needed to confirm this assumption. As mentioned by Solomon et al. in their recent article (13), the concurrent flu and COVID-19 epidemic is a major concern for public health officials and clinicians. Beside effective prevention of flu, this beneficial adjuvant effect of preventing COVID-19 makes flu vaccine an important defence weapon in the approaching threat of new epidemic or pandemic of COVID-19. From a public health perspective, the flu vaccination would prevent the twindemic (flu and COVID-19 pandemics together) (14).

\section{Limitations}

One of the possible limitations of our study is being dependent on the participant's self-declaration of flu vaccination. No confirmatory antibody titre test was done to confirm the vaccination history. Also, we could not include such antibodies data in the analysis. Another limiting point was the presence of 4 flu vaccinated COVID-19 infected participants. So, we could not compare these participants' data with the non-vaccinated COVID-19 infected participants. Also, additional correlation and regression analyses were not possible. But we should mention that this very low COVID-19 cases in the flu vaccinated participant group support the protective role of this vaccine (at least in our patient series). This finding may lead to more detailed studies in this field.

\section{CONCLUSION}

Our pilot study results showed a beneficial effect of seasonal flu vaccination in decreasing the susceptibility to COVID-19 infection. Adding this beneficial adjuvant effect of flu vaccine to its main task of preventing flu will help more in combating COVID-19 outbreak. Still, further detailed studies are needed in this field.

\section{Conflict of Interests}

None declared

\section{Data Availability}

Data are available to researchers (for research purpose only) on request by directly contacting the corresponding author.

\section{REFERENCES}

1. Hibberd PL. Seasonal influenza vaccination in adults [Internet] Up to Date; 2020 [cited 2020 May 5]. Available from: https:// www.uptodate.com/contents/seasonal-influenza-vaccination-inadults? search=influenza vaccine\&source $=$ search_result\&selectedTitle $=2 \sim 144 \&$ usage_type $=$ default\&display_rank $=1$.

2. Li X, Xu S, Yu M, Wang K, Tao Y, Zhou Y, et al. Risk factors for severity and mortality in adult COVID-19 inpatients in Wuhan. J Allergy Clin Immunol. $2020 \mathrm{Jul} ; 146(1): 110-8$.

3. European Commission. EPALE. Newsroom. Successful treatment strategy of Turkey against Covid-19 outbreak [Internet]. Brussels: European Commission; 2020 [cited 2020 Jul 17]. Available from: https://epale. ec.europa.eu/en/content/successful-treatment-strategy-turkey-againstcovid-19-outbreak.

4. Salem ML, El-Hennawy D. The possible beneficial adjuvant effect of influenza vaccine to minimize the severity of COVID-19. Med Hypotheses. 2020 Apr 22;140:109752. doi: 10.1016/j.mehy.2020.109752.

5. Fink G, Orlova-Fink N, Schindler T, Grisi S, Ferrer APS, Daubenberger $\mathrm{C}$, et al. Inactivated trivalent influenza vaccination is associated with lower mortality among patients with COVID-19 in Brazil. BMJ Evid Based Med. 2020 Dec 11;bmjebm-2020-111549. doi: 10.1136/ bmjebm-2020-111549.

6. Thomas L. Research suggests protective effect of influenza vaccine against COVID-19 severity and mortality [Internet]. Manchester: News Medical Life Sciences; 2020 [cited 2020 Jul 21]. Available from: https:// www.news-medical.net/news/20200705/Research-suggests-protectiveeffect-of-influenza-vaccine-against-COVID-19-severity-and-mortality. aspx.

7. Jamovi. Stats. Open. Now. [Internet]. [cited 2020 Jul 21]. Available from: https://www.jamovi.org/.

8. Akoglu H. User's guide to correlation coefficients. Turk J Emerg Med. 2018 Aug 7;18(3):91-3.

9. The National Academies of Sciences, Engineering, and Medicine. Based on science. The flu vaccine does not affect COVID-19 test results or the likelihood of catching COVID-19 [Internet]. Washington: The National Academies of Sciences, Engineering, and Medicine; 2020 [cited 2020 Jul 22]. Available from: https://sites.nationalacademies.org/BasedOnScience/ covid-flu-vaccine/index.htm.

10. Pathak S, Jolly MK, Nandi D. Protective roles of flu infections and BCG vaccination in lowering Covid-19 mortality [Internet]. Durham: Research Square; 2020 [cited 2020 Jul 22]. Available from: https://doi. org/10.21203/rs.3.rs-33242/v1.

11. World Health Organization. Coronavirus disease (COVID-19) advice for the public: Mythbusters [Internet]. Geneva: WHO; 2020 [cited $2020 \mathrm{Jul}$ 
21]. Available from: https://www.who.int/emergencies/diseases/novelcoronavirus-2019/advice-for-public/myth-busters?gclid=CjwKCAjwx9 4BRAHEiwApAt0zrxKB6k9ZvO6zWH-2Ahb1HAnETC66V_HyK41y1U5SsNu8rzfMcgKoxoC6KAQAvD BwE\#vaccines.

12. BBC. News. Brocklehurst S. Why do the majority of NHS staff not get the flu vaccine? [Internet]. BBC; 2018 [cited 2020 Jul 22]. Available from: https://www.bbc.com/news/uk-scotland-42634781.

13. Solomon DA, Sherman AC, Kanjilal S. Influenza in the COVID-19 Era. JAMA. 2020 Oct 6;324(13):1342-3.
14. Paget J, Caini S, Cowling B, Esposito S, Falsey AR, Gentile A, et al. The impact of influenza vaccination on the COVID-19 pandemic? Evidence and lessons for public health policies. Vaccine. 2020 Sep 29;38(42):6485-

Received October 21, 2020 Accepted in revised form February 8, 2021 